\title{
Review on the cervical laminoplasty surgical technique and its clinical employment
}

\begin{abstract}
Cervical laminoplasty as a surgical technique, is used to remove pressure from the cervical spinal cord which has been developed due to various pathologies including herniations of the disc, arthritis, spinal fractures, degenerative changes in the spine, tumors etc. This is a brief review on this surgical technique and its clinical employment.
\end{abstract}

Keywords: cervical laminoplasty, laminoplasty surgical technique
Volume II Issue 6 - 2019

Behzad Saberi
Medical Research, Iran

Correspondence: Behzad Saberi, Esfahan, Iran, Tel 00989131263487, Email sab64b@yahoo.com

Received: October 22, 2019 | Published: December 30, 2019

\section{Introduction}

Various pathologies including tumors, arthritis, disc herniations, degenerative diseases and fractures can affect the cervical spinal cord by causing pressure on it and result in various clinical problems for the affected patients. Removing such pressure from the cervical spinal cord can be done surgically by a technique called cervical laminoplasty. During cervical laminoplasty the volume of the spinal canal is augmented. This surgical procedure is done by a posterior approach to the spine.

Cervical spondylotic myelopathy due to the posterior longitudinal ligament's occification which results in cervical canal stenosis, would be treated by employing laminoplasty surgical technique. Spinal canal's multisegmental stenosis would also be treated by laminoplasty surgical technique. ${ }^{1,2}$

C3 through C7 segments of the spine, are the most common spinal segments which their stenosis would be treated with employing laminoplasty surgical technique. When more than two levels of the spine require decompression, laminoplasty over anterior fusion techniques can be employed. In the recovery rates of the patients with myelopathy, there is no difference between anterior cervical fusion and posterior laminoplasty approach.

Allowing the posterior shift of the spinal cord into the augmented space, makes laminoplasty surgical technique to be an effective treatment method in removing pressure from the cervical spinal cord. Lessening of adjacent segment degeneration, post laminectomy membrane development avoidance, post laminectomy kyphosis prevention and cervical motion preservation, are the benefits of employing laminoplasty surgical technique in theory. ${ }^{3}$ Such results would not be achieved in practice at all times because about 50 percent of the range of motion of the cervical spine, would be decreased after laminoplasty. Also 10 to 35 percent of the patients would develop kyphotic deformity after employing laminoplasty surgical technique. So achieving best surgical results in employing laminoplasty surgical technique is not possible in all of the cases.

Although avoiding the occurrence of fusions after laminoplasty is the treatment goal, but such spontaneous fusions have been reported after laminoplasty in various cases. Inter laminar fusion can be occurred in about 53 percent of the patients after laminoplasty specifically at the $\mathrm{C} 2-\mathrm{C} 3$ level. This cause reduction in the cervical spine's range of motion. Chronic neck pain after laminoplasty specifically in anterior fusions can also occur in about 6 to 60 percent of the patients. ${ }^{4,5}$

The incidence of the cervical spine's adjacent level degeneration, would be decreased by employing laminoplasty surgical technique. Artificial laminar spacers, intra laminar separators, contoured laminoplasty plates and suture anchors as new instrumentation advances, have improved laminoplasty procedures and also caused the laminoplasty procedures to become much more simplified practically. ${ }^{6}$

\section{Conclusion}

Cervical laminoplasty can be an effective surgical technique in removing the pressure from the cervical spine and cause the patient's clinical problems to be solved. It is important for the spine surgeons to have knowledge about this surgical technique to employ it much more effectively during clinical practice.

\section{Acknowledgments}

None.

\section{Conflicts of interest}

The authors declare there are no conflicts of interest.

\section{References}

1. Oyama M, Hattori S, Moriwaki N. A New method of cervical laminoplasty. The Central Japan Journal of Orthopaedic Surgery. 1973;16:792-794

2. Seichi A, Takeshita K, Ohishi I, et al. Long-term results of double-door laminoplasty for cervical stenotic myelopathy. Spine (Phila Pa 1976). 2001;26(5):479-487.

3. Matsunaga S, Sakou T, Nakanisi K. Analysis of the cervical spine alignment following laminoplasty and laminectomy. Spinal Cord. 1999;37(1):20-24.

4. Steinmetz MP, Resnick DK. Cervical laminoplasty. Spine Journal. 2006;6(6): S274-S281.

5. Herkowitz HN. Cervical laminaplasty: its role in the treatment of cervical radiculopathy. J Spinal Disord. 1988;1(3):179-188.

6. Hirabayashi K, Satomi K. Operative procedure and results of expansive open-door laminoplasty. Spine. 1988;13(7):870-876. 